\title{
Genetic and Molecular Analyses indicate independent effects of TGIFs on Nodal and Gli3 in neural tube patterning
}

\author{
Kenichiro Taniguchi ${ }^{1,3}$, Anoush E Anderson ${ }^{1}$, Tiffany A Melhuish ${ }^{1}$, Anne L Carlton ${ }^{1}$, Arkadi Manukyan ${ }^{1}$, \\ Ann E Sutherland ${ }^{2}$ and David Wotton ${ }^{\star 1}$
}

Holoprosencephaly (HPE) is a prevalent craniofacial developmental disorder that has both genetic and environmental causes. The gene encoding TG-interacting factor 1 (TGIF1) is among those that are routinely screened in HPE patients. However, the mechanisms by which TGIF1 variants cause HPE are not fully understood. TGIF1 is a transcriptional repressor that limits the output of the Transforming Growth Factor B (TGFB)/Nodal signaling pathway, and HPE in patients with TGIF1 variants has been suggested to be due to increased Nodal signaling. Mice lacking both Tgif1 and its paralog, Tgif2, have HPE, and embryos lacking Tgif function do not survive past mid-gestation. Here, we show that in the presence of a Nodal heterozygous mutation, proliferation defects are rescued and a proportion of embryos lacking all Tgif function survive to late gestation. However, these embryos have a classic HPE phenotype, suggesting that this is a Nodal-independent effect of Tgif loss of function. Further, we show that the Gli3 gene is a direct target for repression by Tgifs, independent of TGFB/Nodal signaling, consistent with Tgif mutations causing HPE via Nodal-independent effects on the Sonic Hedgehog (Shh) pathway. Based on this work, we propose a model for distinct functions of Tgifs in the Nodal and Shh/Gli3 pathways during forebrain development.

European Journal of Human Genetics (2017) 25, 208-215; doi:10.1038/ejhg.2016.164; published online 7 December 2016

\section{INTRODUCTION}

Holoprosencephaly (HPE) is a severe developmental disorder affecting forebrain and facial development. ${ }^{1,2}$ In humans, HPE affects approximately $1 / 8000$ live births and up to $1 / 250$ conceptuses, making it the most frequent forebrain developmental disorder in humans. ${ }^{3}$ The primary defect in HPE is a failure of the ventral forebrain to divide into two hemispheres, and this is associated with defects in midline facial structures. ${ }^{4,5}$ Of the known HPE loci, those that encompass the SHH, ZIC2, SIX3 and TGIF1 genes are most commonly screened as part of routine genetic evaluation of HPE patients. Best understood in terms of HPE causation are variants affecting the $S H H$ gene, which encodes Sonic Hedgehog, a secreted morphogen with a wide range of developmental functions. ${ }^{6,7}$ Heterozygous loss of function $\mathrm{SHH}$ variants account for $17 \%$ of familial HPE and $3.7 \%$ of sporadic cases. ${ }^{8-10}$ Although heterozygous Shh mutant mice are normal, homozygous mutants are inviable, and have HPE in utero. ${ }^{10,11}$ Expression of Shh in the ventral diencephalon helps specify ventral identity and limits expression of the Gli3 gene. Gli3, which is primarily expressed dorsally, promotes dorsal fate and inhibits the ventral Shh signal, ${ }^{12}$ and Gli3 null embryos have a forebrain with dorsally expanded ventral tissue. ${ }^{13,14}$ Homozygous Shh null embryos have a forebrain ventricle that lacks ventral identity and fails to divide into two hemispheres. ${ }^{11}$ Introduction of Gli3 mutations into Shh null embryos results in partial rescue of ventral identity, suggesting antagonism between Shh and Gli3 in dorsoventral (D-V) patterning.
The Thymine-Guanine Interacting Factorl gene (TGIF1) was identified in the minimal critical region of the HPE4 locus, with TGIF1 variants in HPE patients being heterozygous loss of function mutations. ${ }^{15,16}$ There is no evidence for HPE-associated variants in the human TGIF2 gene, but in mice Tgif1 and Tgif2 share overlapping function during development. ${ }^{17,18}$ Homozygous deletion of either Tgif1 or Tgif2 does not cause severe phenotypes in mice. ${ }^{17,19-22}$ However, embryos lacking both genes fail gastrulation, with defects in the Nodal signaling pathway. ${ }^{17}$ Epiblast-specific deletion of a conditional Tgif1 allele in a Tgif2 null background allows for bypass of the gastrulation defects, and these conditional double null ( $\mathrm{cdKO})$ embryos survive to $\sim 11.0 \mathrm{dpc}$ with precursor forms of HPE similar to those seen in Shh null embryos. ${ }^{17,18}$ Although changes the SHH pathway in the absence of Tgifs have been documented, it is not known whether this is dependent on Nodal signaling.

TGIF1 and TGIF2 are homeodomain-containing transcriptional repressors that interact with mSin3 and HDACs via a conserved repression domain close to their carboxyl-termini. ${ }^{23-26}$ TGIF1 also interacts with the CtBP transcriptional corepressors via its amino terminus. ${ }^{27}$ TGIF1 and TGIF2 are transcriptional corepressors for Transforming Growth Factor (TGF) B-activated Smads. ${ }^{28-30}$ In response to TGFß, Activin or Nodal, Smad2 and Smad3 are phosphorylated, complex with Smad4, and accumulate in the nucleus to activate gene expression. ${ }^{31,32}$ TGIF1 and TGIF2, limit gene expression by competing with coactivators and recruiting general corepressors to the Smads. In mouse embryos lacking both Tgifl and

${ }^{1}$ Department of Biochemistry and Molecular Genetics, and Center for Cell Signaling, University of Virginia, Charlottesville, VA, USA; ${ }^{2}$ Department of Cell Biology, University of Virginia, Charlottesville, VA, USA

*Correspondence: Dr D Wotton, Center for Cell Signaling, University of Virginia, Box 800577, HSC, Charlottesville, VA 22908, USA. Tel: +1 4342436752 ; Fax: +1 434924 1236; E-mail: dw2p@virginia.edu

3Present address: Department of Cell and Developmental Biology, University of Michigan Medical School, Ann Arbor, MI 48109, USA.

Received 13 May 2016; revised 14 October 2016; accepted 25 October 2016; published online 7 December 2016 
Tgif2, gene expression changes, left-right asymmetry and forebrain defects can be partially ameliorated by introducing a heterozygous Nodal mutation, consistent with the role of Tgifs in this pathway. ${ }^{17,18}$ TGIF1 and TGIF2 can also bind directly to DNA, and a small number of direct TGIF1 target genes have been characterized. ${ }^{33-35}$ While it is likely that TGIF1 and TGIF2 modulate gene expression by multiple mechanisms, it is not known how they regulate expression of genes involved in HPE pathogenesis.

Here we show that Tgifl binds directly to a conserved element in the Gli3 gene to repress Gli3 expression, independent of TGFß/Nodal. In contrast, defective neuroepithelial cell proliferation in $\mathrm{cdKO}$ embryos, is rescued by Nodal heterozygosity. A proportion of Nodal heterozygous cdKO embryos survive to $18.5 \mathrm{dpc}$, but have classic HPE phenotypes, consistent with a failure to rescue the Gli3-Shh balance. This work shows a separation of function for the Nodal and Shh pathways in the pathogenesis of HPE, and suggests that HPE in patients with TGIF1 variants may not be caused by increased Nodal signaling as previously thought. ${ }^{16}$

\section{MATERIALS AND METHODS}

Mice

All animal procedures were approved by the Animal Care and Use Committee of the University of Virginia, which is fully accredited by the AAALAC. The Tgifl allele, (exons 2 and 3 flanked by loxP sites), ${ }^{22}$ Tgif 2 null (deletion of the first coding exon), ${ }^{17}$ the Gli3 allele (loxP sites flanking exon 9) and Nodal mutants (a ß-gal insertion into the first coding exon) are as described. ${ }^{18}$ Deletion was generated using Sox $2 \mathrm{Cre},{ }^{36}$ which is active in the epiblast after $\sim 5.5 \mathrm{dpc}$. MEFs were isolated as in, ${ }^{30}$ from wild-type or Tgiflf;; Tgif2 ${ }^{-/}$; $R 26 \mathrm{CreER}$ embryos. Recombination was induced by two $16 \mathrm{~h}$ treatments with $100 \mathrm{~nm}$ 4-hydroxy-Tamoxifen, each followed by $8 \mathrm{~h}$ with fresh medium. All mouse lines were maintained on a mixed C57BL/6 J $\times 129 \mathrm{~Sv} / \mathrm{J}$ background. Genomic DNA for PCR genotyping was purified from ear punch, at post-natal day 21 (P21), yolk sac $(7.0-10.0 \mathrm{dpc})$, or tail $(18.5 \mathrm{dpc})$ by HotShot. Phenotype data has been submitted to the Mouse Genome Informatics database (http:// www.informatics.jax.org/).

\section{In situ hybridization}

Whole-mount in situ hybridization was performed with digoxigenin-labeled riboprobes, as described. ${ }^{18}$ All images are representative of at least three embryos analyzed.

Histology, immunohistochemistry (IHC) and whole-mount analysis Embryos were fixed overnight in $4 \%$ paraformaldehyde at $4{ }^{\circ} \mathrm{C}$, or for $48 \mathrm{~h}$ for $18.5 \mathrm{dpc}$, dehydrated, sectioned at $7 \mu \mathrm{m}$, and stained with Hematoxylin and Eosin (H\&E) as described. ${ }^{18}$ Sections were incubated with antibodies against phosopho-histone H3 (1:200, Upstate 06-570), and imaged as described previously. ${ }^{17,37}$ The percentage of $\mathrm{pHH} 3$ positive nuclei were counted in the forebrain neuroepithelium (NE) from three embryos per genotype from at least two sections per embryo. Data were compared using a Student's $T$ test. Antibody staining was detected using Vectastain $\mathrm{ABC}$ (Vector Laboratories, Burlingame, CA, USA) and developed with Impact DAB (Vector Laboratories). Images were captured using an Olympus BX51 microscope and either an Olympus SZX12 or DP70 digital camera, and processed in Adobe Photoshop. Whole-mount images were captured using a Leica MZ16 stereomicroscope and QImaging 5.0 RTV digital camera.

\section{Chromatin immunoprecipitation and DNA affinity precipitation}

ChIP was performed as previously described. ${ }^{38}$ Cells were cross-linked with $1 \%$ formaldehyde for $20 \mathrm{~min}$ at $37^{\circ} \mathrm{C}$. Following chromatin isolation, DNA was sheared to 200-1000 bp using a Branson digital sonifier, with microtip. For ChIP from whole embryos, freshly isolated tissue was gently dissociated and cross-linked for $20 \mathrm{~min}$ at $37^{\circ} \mathrm{C}$. Immunoprecipitations were carried out using $2-10 \mu \mathrm{l}$ of a polyclonal Tgif1 antiserum, ${ }^{29}$ preimmune serum, or a Smad2/3specific antiserum. Bound and input fractions were analyzed by qPCR using a
BioRad MyIQ cycler and Sensimix Plus SYBRgreen plus FITC mix (Bioline, Taunton, MA, USA). DNA affinity precipitation was performed as described, ${ }^{38}$ using biotinylated double-stranded oligonucleotides corresponding to the putative TGIF site from the human GLI3 HCNR1, or a mutant version of it. Bound proteins were identified by western blot.

\section{siRNA knock-down and qRT-PCR}

RNA was isolated and purified using Absolutely RNA kit (Agilent, Cedar Creek, TX, USA). cDNA was generated using Superscript III (Invitrogen, Grand Island, NY, USA), and analyzed in triplicate by real time qPCR, with intron spanning primer pairs, selected using Primer3 (http://frodo.wi.mit.edu/). Expression was normalized to Rpl4 and Actin using the delta Ct method. For knock-down, cells were plated in 6 well plates and transfected with Dharmacon SMARTpool oligonucleotides against TGIF1 and TGIF2, using DharmaFECT reagent 1 .

\section{Cell culture and luciferase assays}

U87 and A172 cells were grown in DMEM with 10\% FBS, and transfected using TurboFect (ThermoFisher, Waltham, MA, USA) according to the manufacturer's instructions. Primary MEFs were isolated from 13.5 day mouse embryos, and cultured in DMEM (Invitrogen) with 10\% Fetal Bovine Serum (Hyclone; GE Healthcare Lifesciences, Logan, UT, USA) as described. ${ }^{30}$ Luciferase assays were performed as described, ${ }^{38}$ with normalization to a cotransfected Renilla control plasmid.

\section{RESULTS}

\section{Gli3 is a direct Tgif target gene}

We have previously shown that embryos lacking both Tgif1 and Tgif2 have HPE and defects in the Shh signaling pathway. ${ }^{18}$ We observed a partial rescue of the forebrain defects by introducing a heterozygous Nodal mutation. Reducing Gli3 expression, which was increased in cdKO embryos compared with wild-types, also resulted in a partial phenotypic rescue. One possible model is that increased signaling from Nodal causes increased Gli3 expression and a decrease in Shh expression in the ventral region of the forebrain. To test this model, we first examined expression of Gli3 in cdKO embryos and in cdKO embryos with a mutation in the Nodal gene. At 9.0 days post coitum $(\mathrm{dpc})$ we observed an increase in dorsal expression of Gli3 in cdKO embryos, but this increase was not reversed by a heterozygous Nodal mutation (Figure 1a).

Since we observed no effect of Nodal mutation on Gli3 expression, we tested whether Gli3 could be regulated directly by Tgifs. Several conserved non-coding elements have been identified within the Gli3 gene, which spans $>250 \mathrm{~kb}$ in both mice and humans. ${ }^{39,40}$ Additionally, in a ChIP-seq experiment using antibodies against the coactivator, p300, a number of conserved forebrain-specific peaks were identified. ${ }^{41}$ We scanned these conserved regions for consensus Tgif binding sites (CTGTCA) present in both the mouse and human sequences, and identified seven putative Tgif sites in five of these conserved regions (Figure 1b). There were also matches to the minimal Smad binding element (CAGA) in each region, but given that this is only a four base consensus, it is expected at a higher frequency. We first tested binding by performing ChIP-qPCR for each of the Tgif sites using chromatin isolated from $9.0 \mathrm{dpc}$ wild-type embryos. Chromatin was precipitated with a Tgifl antiserum, the preimmune serum or a Smad2/3-specific antiserum. As shown in Figure $1 \mathrm{c}$, we observed robust enrichment of the HCNR1 region ${ }^{40}$ in the Tgifl precipitates, whereas no other regions tested were bound by Tgifl. No binding of Smad2/3 was observed to any of the regions tested. To confirm this, we examined binding of Tgifl to the HCNR1 and to two of the other regions for comparison, in NIH3T3 cells and in primary mouse embryo fibroblasts (MEFs). In NIH3T3 cells, Tgif1 


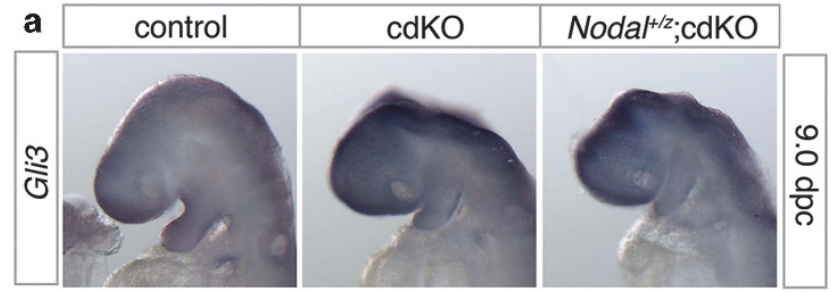

b

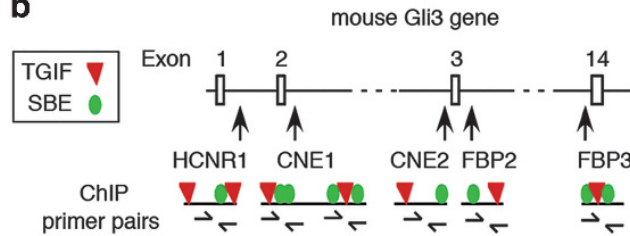

C

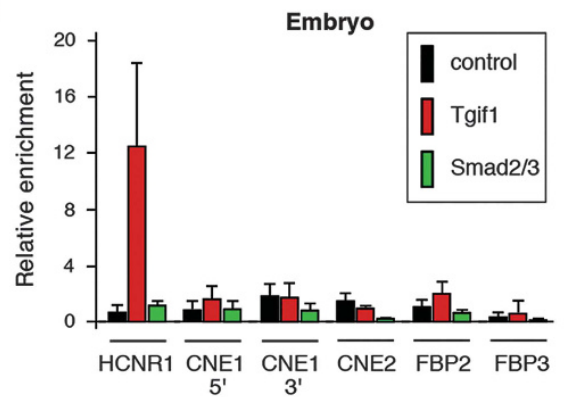

d

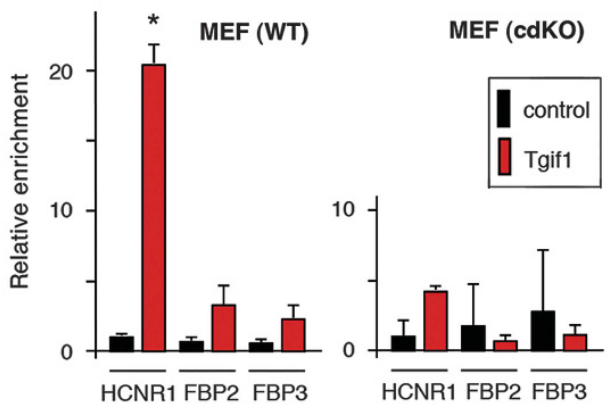

e


f



g



h

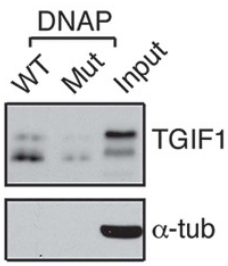

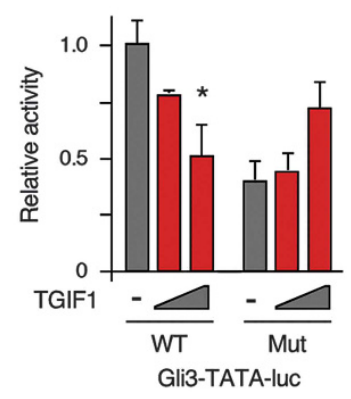

j

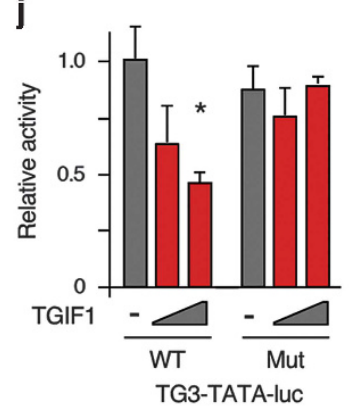

Mm CAAATCAGAA CTGTCACTCAGGGCCG

||||||||||||||||||||||

Hs CAAATCAGAA CTGTCA CTCAGGGCTT

Mut CGTCAA

Figure 1 Gli3 is a Tgif1 target gene. (a) Stage matched control and cdKO embryos, and cdKO embryos with heterozygous Nodal (Nodal/z; cdKO) mutations, at $9.0 \mathrm{dpc}$, were analyzed by in situ hybridization with an anti-sense probe for Gli3. Embryos are representative of at least three in each case. (b) A truncated version of the Gli3 gene is shown schematically, with exons 1-3 and 14 indicated, together with the conserved regions shown below. Putative TGIF sites and Smad binding elements (SBE) are shown. (c) Binding of TGIF1 to conserved non-coding regions from the Gli3 gene was analyzed by ChIP-qPCR in wild-type 9.0 dpc embryos. Chromatin was precipitated with a Tgif1-specific rabbit antiserum, a Smad2/3 specific serum or a control nonimmune serum. (d) Primary wild-type and cdKO MEFs were analyzed in triplicate by ChIP and GPCR using the Tgif1-specific serum or control. Relative binding compared with pre-immune serum is shown. ${ }^{*} P<0.001$ by student's $T$ test. (e) U87 and A172 human glioma cell lines were transfected with siRNAs against TGIF1 and TGIF2 or a control pool, and analyzed by qRT-PCR. Relative expression of TGIF1, TGIF2 and GLI3 is shown. ${ }^{*} P<0.05$ by student's $T$ test. (f) Expression of the indicated genes was analyzed by qRT-PCR in U87 cells treated with TGFß for 3 or $9 \mathrm{~h}$ or left untreated. *P<0.01 by student's $T$ test, compared with untreated control. (g) Binding of TGIF1 to the human HCNR1 was analyzed by ChIP and qPCR in chromatin from U87 cells. ${ }^{*} P<0.01$ by student's $T$ test. (h) Binding of TGIF1 from U87 cells to a wild-type or mutated version of the HCNR1 TGIF consensus site was analyzed by DNA affinity precipitation, followed by western blotting for TGIF1, and tubulin as a control. (i, j) Relative luciferase activity is shown for two reporters based on the TGIF site in HCNR1, in which the TGIF site was intact (WT) or mutated (Mut). The sequence surrounding the site, together with the mutant is shown below. ${ }^{*} P<0.01$ by student's $T$ test, compared with no TGIF1 control.

again bound the HCNR1 region, and binding was not affected by the addition of TGFß for one hour prior to isolating the chromatin (Supplementary Figure S1A). Smad2/3 binding to HCNR1 was not observed and addition of TGFß did not change expression of the endogenous Gli3 gene in NIH3T3 cells (Supplementary Figure S1). To ensure that the observed binding was indeed due to Tgifs, we next tested primary MEFs that were either wild-type, or lacked both Tgif1 and Tgif2 expression. cdKO MEFs were generated using a Tamoxifen 
inducible Cre transgene and the Tgif1 conditional allele in the background of a Tgif2 null. We chose to test cdKO MEFs rather than Tgif1 single null MEFs as the Tgif1 antiserum may cross-react with Tgif2. In wild-type MEFs, the HCNR1 region was highly enriched in Tgif1 precipitates, whereas we observed minimal binding to the other two regions tested and no significant enrichment for any of the three regions tested in chromatin from cdKO MEFs (Figure 1d).

To test whether Tgifs regulate GLI3 expression in a different system, we used human glioblastoma cell lines and tested effects of transient knock-down of TGIF1 and TGIF2. U87 cells express relatively high levels of TGIF1, and both TGIF1 and TGIF2 are highly expressed in A172 cells. Additionally, both cell lines express endogenous GLI3. U87 and A172 cells were transfected with siRNA pools targeting both TGIF1 and TGIF2 or with a control pool and RNA was analyzed by qRT-PCR $72 \mathrm{~h}$ later. As shown in Figure 1e, we obtained at least 75\% knock-down of each gene, and also observed a significant increase in GLI3 expression in both cell lines. As in NIH3T3 cells, we did not observe any induction of GLI3 expression by TGFß signaling in U87 cells (Figure 1f). However, we were able to detect TGIF1 at the human HCNR1 region by ChIP using chromatin from U87 cells (Figure 1g). These data suggest that Tgifs are able to bind to a conserved region of the GLI3 gene, and that GLI3 expression is repressed by Tgifs. To determine whether the conserved TGIF consensus site present within HCNR1 was responsible for TGIF1 recruitment and repression of GLI3 expression we examined binding of endogenously expressed
TGIF1 to a double-stranded oligonucleotide encompassing this site by DNA affinity precipitation and western blot. TGIF1 from both U87 and A172 glioblastoma cell lines bound to the wild-type HCNR1 oligonucleotide, whereas binding was clearly reduced with the mutant sequence (Figure 1h, Supplementary Figure S2). To test whether TGIF was able to repress via this sequence we created two transcriptional reporters: one in which an $\sim 100 \mathrm{bp}$ region surrounding the putative TGIF site from HCNR1 was placed upstream of a minimal TATA element (Gli3-TATA-luc), and one containing three copies of the TGIF site (TG3-TATA-luc). As shown in Figure 1i, co-expression of TGIF1 resulted in significant repression of activity from the Gli3TATA-luc reporter, but was unable to repress a mutant version in which the TGIF site had been abolished. Similar results were obtained with the second reporter containing three copies of either the wildtype TGIF site or a mutant version (Figure 1j). Taken together, these data suggest that TGIF1 binds directly to a conserved consensus site in human and mouse GLI3 to repress gene expression independent of TGFß signaling.

\section{Analysis of phenotypes in cdKO embryos with Nodal and Gli3 mutations}

Examination of Shh expression revealed a clear decrease in expression in the ventral region of the forebrain in cdKO embryos, and at $9.0 \mathrm{dpc}$ we did not see any obvious restoration of normal expression with mutations in Gli3, Nodal or both together (Figure 2a). However, when


Figure 2 Shh expression in cdKO embryos. Stage matched control and cdKO embryos, and cdKO embryos with heterozygous Nodal (Nodal+/z;cdKO) or heterozygous Gli3 $\left(\mathrm{Gli} 3^{+/ r} ; \mathrm{cdKO}\right)$ mutations, or with both together $\left(G 3^{+/ r} ; \mathrm{N}^{+/ z} ; \mathrm{cdKO}\right)$ were analyzed by in situ hybridization with an anti-sense probe for Shh, at 9.0 (a) and 9.5 (b) dpc. Side and front views of the same embryos are shown in $\mathbf{b}$. Embryos are representative of at least three in each case. In b, four of five $\mathrm{G3}^{+/ r} ; \mathrm{N}^{+/ z} ; \mathrm{cdKO}$ embryos had the phenotype shown. 
we examined embryos approximately half a day later, at $9.5 \mathrm{dpc}$, we observed a partial restoration of Shh expression in the ventral forebrain in cdKO embryos lacking one copy each of both Gli3 and Nodal (Figure 2b, arrows). In contrast, cdKO embryos with mutations in either Gli3 or Nodal alone had no detectable Shh expression in the ventral forebrain.

To further test the effect of reducing both Nodal and Gli3 on forebrain development we examined the overall structure of the forebrain ventricle. As shown in Figure 3a, in wild-type embryos at $9.5 \mathrm{dpc}$, the NE cell layer bisects the underlying mesenchyme at the midline (arrow, Figure 3a). In stage-matched cdKO embryos there is a much thicker mesenchyme layer ventral to the NE (bracket, Figure 3a). Deletion of Gli3 improved the overall shape of the NE, whereas the Nodal heterozygotes still had a very thick ventral mesenchyme layer. In the double heterozygous (Gli3;Nodal) cdKO embryos, overall ventral patterning was improved with the mesenchyme bisected by the NE (Figure 3a). To examine effects on facial patterning, we tested expression of the eye-field marker, $P a x 2 .{ }^{42}$ The normal robust expression in the two eye-fields at $9.75 \mathrm{dpc}$ was reduced to a single much weaker band of expression in cdKO embryos (Figure 3b). In the Gli3 and the Nodal heterozygous cdKO embryos, expression of Pax2 was somewhat increased, but did not fully separate into two distinct domains, as seen in the wild-type. However, in the Gli3;Nodal double heterozygotes expression was stronger and fully separated into two domains, suggesting improved facial patterning in these embryos (Figure 3b).

We have previously shown that cdKO embryos have a reduced mitotic index in the forebrain NE. ${ }^{18}$ It is possible that decreased proliferation might contribute to the apparent failure to separate the two eye-fields in the cdKO. To test whether the reduced proliferation was due to altered Nodal signaling or reduced Gli3 expression, we stained forebrain sections for phospho-histone $\mathrm{H} 3$ ( $\mathrm{pHH}$ ) to identify

a
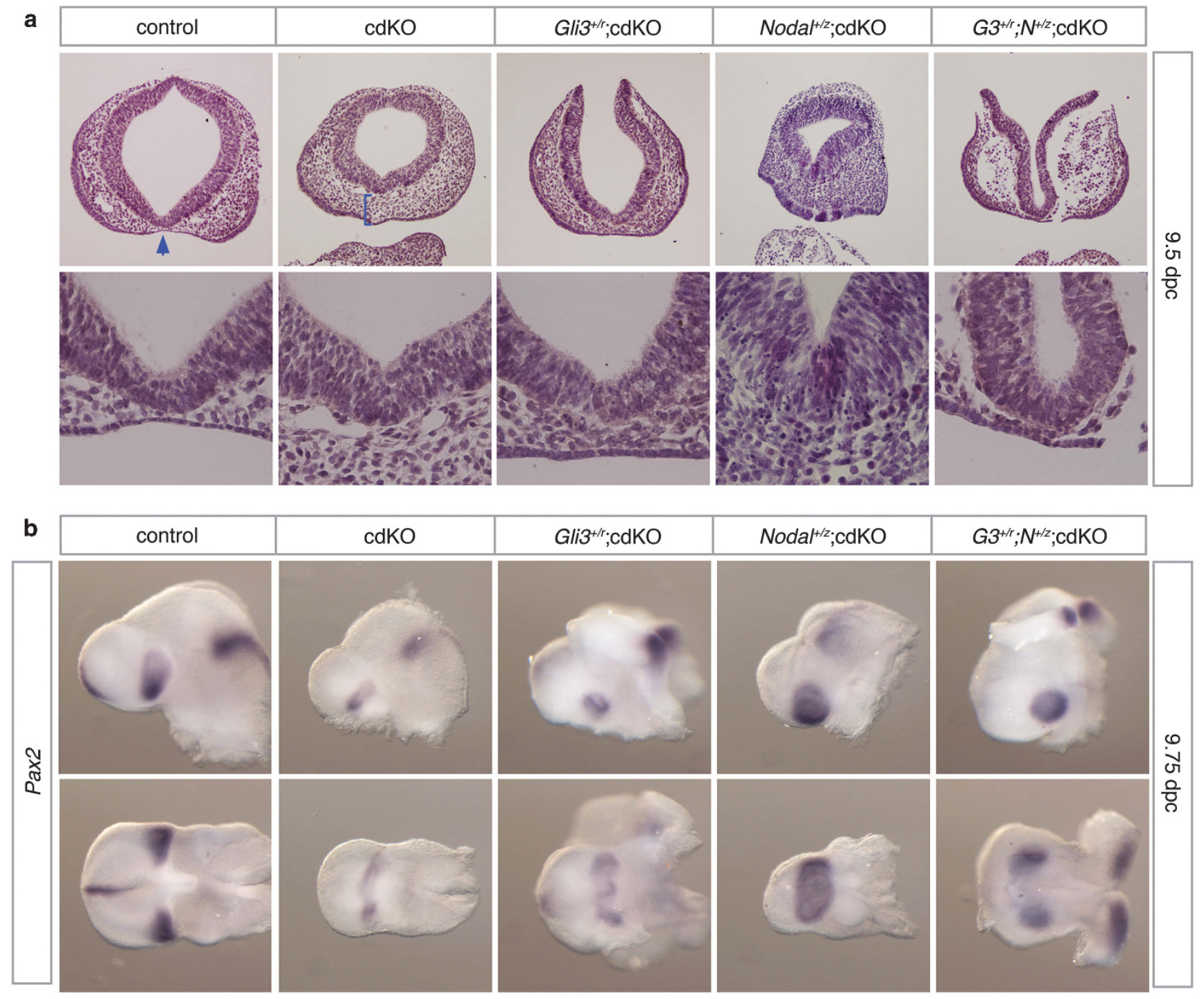

Figure 3 Phenotypic rescue by Nodal and Gli3 mutations. (a) Stage matched embryos of the indicated genotypes, at $\sim 9.5 \mathrm{dpc}$, were analyzed by H\&E staining. A section through the forebrain is shown, together with a higher magnification view of the ventral region. (b) Embryos of the indicated genotypes were analyzed by in situ hybridization with an anti-sense probe for Pax2. A side view of the head is shown, with a ventral view shown below. Arrow in a indicates bisection of the mesenchyme by the neuroepithelial layer, resulting in separation of the presumptive eye-fields. Bracket indicates the thickened mesenchyme in the cdKO. 
Table 1 Neuroepithelial mitotic index

\begin{tabular}{lcc}
\hline Genotype & $\%$ pHH3: mean (sd) & P value vs wild-type \\
\hline Wild-type & $10.02(2.03)$ & \\
cdKO & $3.01(1.90)$ & 0.0119 \\
cdKO;Nodal+/z & $10.39(1.32)$ & 0.8038 \\
cdKO;Gli3+/r & $3.20(1.02)$ & 0.0065 \\
\hline
\end{tabular}

cells in late G2 and M phases of the cell cycle. As shown in Table 1, the mitotic index of the cdKO forebrain NE was significantly lower than that seen in the wild-type embryos. In agreement with previous work, deletion of one copy of Gli3 did not affect the mitotic index. However, removing one copy of Nodal restored the cdKO mitotic index to close to that observed in wild-type embryos (Table 1). This analysis suggests that in the $\mathrm{cdKO}$ forebrain NE there is reduced proliferation and that this phenotype is downstream of excess Nodal signaling. Taken altogether, these data suggest that loss of Tgif function may affect forebrain development by two independent pathways, one via increased Gli3 expression and one via an increased Nodal response.

\section{HPE in late-stage cdKO embryos with Nodal heterozygous mutations}

cdKO embryos do not generally survive beyond $11.0 \mathrm{dpc}$, although from a large number of litters (118 embryos) isolated at $12.5 \mathrm{dpc}$, we did identify two cdKO embryos with cyclopia and clear signs of HPE. ${ }^{18}$ However, both these embryos were severely defective and would likely not have survived much beyond this stage. To test whether reduced Nodal signaling might allow cdKO embryos to survive to later in embryogenesis, we isolated embryos at $18.5 \mathrm{dpc}$ from crosses in which one fourth of the embryos were expected to be cdKO and half of those should be heterozygous for Nodal. As shown in Table 2, we recovered two cdKO embryos at this stage, both of which were also heterozygous for Nodal. In contrast, we did not identify any cdKO embryos with only wild-type Nodal alleles from this analysis, or from other crosses lacking the Nodal heterozygous mutation in one of the parents. Embryos with either one, two or three wild-type alleles of Tgif1 or Tgif2 were found at approximately the expected frequencies, with slightly fewer having a Nodal heterozygous mutation than those with two wild-type alleles (Table 2). Among the 53 embryos in this analysis we identified several with severe phenotypes, including three with a classic HPE phenotype. Interestingly, both of the Nodal heterozygous cdKO embryos, as well as one three-allele mutant that was also heterozygous for Nodal, had HPE (Table 2 and Figure $4 \mathrm{a}$ and b). The other clearly defective embryos were also Nodal heterozygous three-allele mutants, two having exencephaly and one with agnathia. This analysis suggests that reducing the excess Nodal signaling in cdKO embryos allows defective embryos to develop to late embryogenesis, but does not rescue the HPE phenotype. For comparison, we also analyzed 78 embryos at 18.5 dpc from crosses including a Gli3 mutation. However, we did not identify any cdKO embryos among these litters, either with or without a Gli3 heterozygous mutation. From this analysis approximately 17 Gli3 heterozygous cdKO embryos would have been expected. Among the 26 Gli3 heterozygotes with three mutant alleles of Tgif1/Tgif2, half had exencephaly, but we did not observe any with HPE-like phenotypes (Table 2). This is consistent with a model in which direct repression of Gli3 by Tgifs maintains anterior $\mathrm{D}-\mathrm{V}$ patterning, whereas limiting Nodal signaling allows for normal cell proliferation. With deregulated Nodal and Gli3 signaling, cdKO embryos develop a
Table 2 Phenotype analysis of 18.5 day embryos

\begin{tabular}{lcccc}
\hline & $\begin{array}{c}\text { cdKO } \\
\text { Nodal+/z }\end{array}$ & & $\begin{array}{c}\text { 3 allele mutant } \\
\text { Nodal+/z }\end{array}$ & Gli3+/r \\
\hline HPE & 2 & 1 & 1 & \\
Exencephaly & & & 1 & 13 \\
Agnathia & & & & 1 \\
Arrest & & & & 1 \\
Resorbing & 2 & 42 & 40 & 11 \\
Normal & 100 & 2.3 & & 58 \\
Total & & & & \\
\% Defective & & &
\end{tabular}
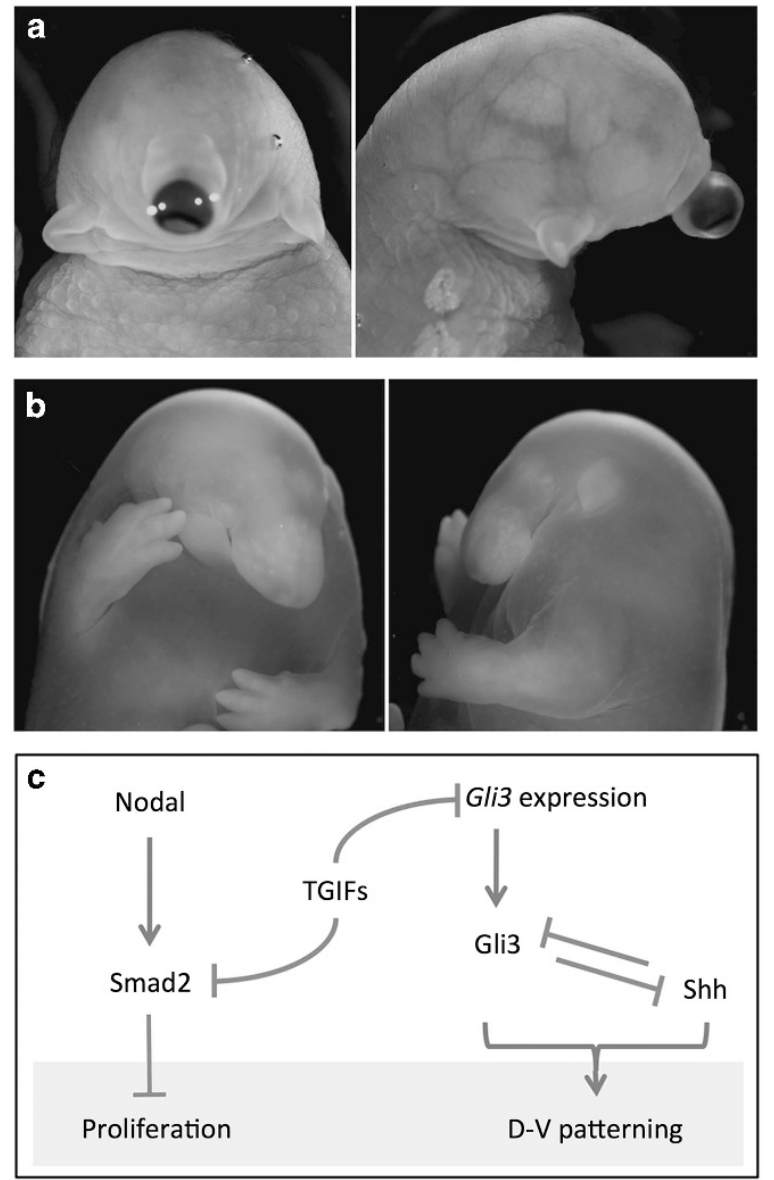

Figure $4 \mathrm{HPE}$ in Nodal heterozygous cdKO embryos at late gestation. (a, b) Examples of whole-mount images of Nodal heterozygous cdKO embryos are shown. (c) A tentative model for the function of Tgifs in forebrain development is shown: TGIFs regulate Gli3 gene expression and the transcriptional activity of Smad2, in the Nodal pathway via independent mechanisms. Arrows indicate a positive effect, lines with cross-bars indicate an inhibitory effect. See text for further discussion.

precursor HPE-like phenotype, but die by approximately $11 \mathrm{dpc}$. Reducing Nodal signaling in this context can restore cell proliferation, but the excess Gli3 expression still causes HPE. This work establishes that Tgifs regulate forebrain development by two independent pathways (Figure 4c). 


\section{DISCUSSION}

In mice loss of Tgif1 and Tgif2 together causes HPE and disrupts Nodal and Shh signaling. ${ }^{18}$ Here we show that Tgifs repress Gliz gene expression and that excess Gli3 contributes to patterning defects in cdKO embryos. In contrast, excess Nodal signaling in the absence of Tgifs causes defects in NE cell proliferation, consistent with two independent pathways causing the forebrain defects observed in the absence of Tgif function (Figure 4c). Importantly, this suggests that the HPE phenotypes seen in patients with TGIF1 variants are due to excess Gli3 expression, but are not as previously suggested ${ }^{16}$ downstream of excess Nodal signaling.

We have previously shown that embryos lacking both Tgifs have phenotypes consistent with an early precursor form of HPE and at these early stages appear quite similar to Shh null embryos. ${ }^{18}$ The majority of cdKO embryos do not survive past $\sim 11.0 \mathrm{dpc}$, so it is difficult to examine later phenotypes that are more clearly representative of HPE. In previous analyses we did identify two cdKO embryos at $12.5 \mathrm{dpc}$. Both embryos had cyclopia and an anterior proboscis-like structure, as seen in Shh null embryos at this age. However, these embryos were quite rare and were very unlikely to survive beyond this stage. From a smaller cohort of embryos analyzed here at $18.5 \mathrm{dpc}$, we found three with clear HPE, including cyclopia and proboscis. Two of these embryos were cdKOs and one had three mutant alleles (Tgif1 null; Tgif2 heterozygous). The presence of an HPE phenotype in a three-allele mutant is consistent with previous work suggesting that a small proportion $(5-10 \%)$ of embryos of this genotype are indistinguishable from cdKOs at $9.0-10.5 \mathrm{dpc}$. What is most striking from the analysis here is that all three embryos with HPE, including the only two cdKO embryos identified at $18.5 \mathrm{dpc}$, were also heterozygous for Nodal. In contrast, we did not identify any Gli3 heterozygous cdKO embryos at this late-stage of embryogenesis, and none of the Gli3 heterozygous three-allele mutants had HPE. One explanation for this is that Nodal heterozygosity can rescue the proliferation defects seen in cdKO embryos but not the anterior $\mathrm{D}-\mathrm{V}$ patterning defects. In cdKOs where Nodal heterozygosity allows survival to late embryogenesis we observe a classic HPE phenotype which is likely caused by the excess Gli3 expression earlier in development. Thus it is possible that Tgif mutations cause HPE, not by disrupting Nodal signaling as initially thought, but rather by increasing Gli3 expression and disrupting Shh signaling independent of Nodal signaling. The introduction of a Gli3 mutation into cdKO embryos did improve forebrain $\mathrm{D}-\mathrm{V}$ patterning, but was insufficient to fully rescue the phenotype. This lack of complete rescue could simply be due to the half dose of Gli3 not reaching the appropriate level for normal development or could imply some contribution of other pathways. Embryos that were doubly heterozygous for both Nodal and Gli3 appeared to have further improved ventral patterning, although they also had severe defects dorsally. This suggests that the effects of reducing Gli3 are more apparent when overall NE integrity is improved, although it remains possible that excess Nodal signaling may also affect other aspects of forebrain patterning.

The HPE observed at $18.5 \mathrm{dpc}$ in Nodal heterozygous cdKO embryos argues against a direct role for excess Nodal signaling in causing HPE. Previous studies have suggested that mutations that reduce the activity of the Nodal/Smad pathway can cause HPE. ${ }^{43}$ This has seemed somewhat at odds with the HPE observed in patients with heterozygous TGIF1 variants and in our cdKO embryos where loss of Tgifs would be expected to increase Smad2 transcriptional output in response to Nodal. In a previous analysis of Smad2;Nodal mutants we observed primarily anterior truncations, and only one Smad2;Nodal double heterozygous embryo with HPE-like phenotypes. ${ }^{18}$ While these truncations can appear similar to the proboscis-like phenotype seen with HPE, the tissue is clearly distinct with most of the presumptive forebrain tissue being absent. Evidence from human studies does suggest that reduced Nodal signaling can contribute to HPE. Variants in the NODAL, FOXH1 and TDGF1 genes have all been found in human HPE patients and it is likely that these are reduced function variants. ${ }^{44,45}$ However, it should be noted that these variants are quite rare in HPE and are more generally associated with other congenital defects. The work here raises the possibility that in HPE patients with loss of function variants in these genes, reduced Nodal pathway activity may contribute to HPE by uncovering effects of additional variants.

Previous analyses have shown that reducing Gli3 dosage in the background of a Shh null mutation can partially rescue the defects in $\mathrm{D}-\mathrm{V}$ patterning and the HPE phenotype. ${ }^{12}$ Based on our analysis here and in previous work, it appears that Gli3 heterozygosity partially rescues $\mathrm{D}-\mathrm{V}$ patterning and HPE in the context of loss of Tgif function. This is clearly consistent with the HPE phenotypes in cdKO embryos being due to altered Shh pathway activity. The increase in Gli3 expression in cdKO embryos appears to be independent of the TGFß/Nodal pathway. Since Tgifs are transcriptional repressors, direct target genes would be expected to have higher expression in the cdKO than in wild-type embryos, and we show that Tgifl binds to a conserved region of the Gli3 gene via a conserved consensus Tgif site. Several studies have focused on conserved non-coding regions of the Gli3 gene with the goal of identifying important regulatory elements. A large number of conserved regions have been identified and several have been characterized for transcriptional activity in chick or mouse. ${ }^{39}$ The region to which we show Tgifl binding has previously been shown to direct reporter expression in chick neural tube and to be bound by homeodomain proteins of the $\mathrm{Pbx}$ and Meis/Prep families. ${ }^{40}$ Here we show that Tgifl is also able to bind directly to this element and this results in transcriptional repression, at least in mouse embryos and human glioblastoma cell lines. However, given the complexity of the regulatory elements that have been identified within the Gli3 locus, ${ }^{39,40}$ it is possible that in other cell types regulation by Tgifs is less important, or that TGFß signals play a role in regulating Gli3 expression. However, our analysis suggests that at least in some contexts Tgifs regulate Gli3 expression independent of TGFß family signaling. At some genes, Tgif1 and Meis family proteins have been shown to compete for binding to the same sites to either repress or activate gene expression. ${ }^{33,35}$ This raises the possibility that competition between Tgifs and other homeodomain proteins may fine-tune Gli3 expression levels at some stages of development.

In summary, we show that in embryos lacking Tgif function, both the Nodal and Shh signaling pathways are disrupted and that these appear to be independent of each other (Figure 4c). Our genetic analysis suggests a context in which variants that reduce TGFß/Nodal signaling could contribute to the pathogenesis of HPE, and we propose a model for the involvement of Tgif function in Nodal and Shh signaling HPE.

\section{CONFLICT OF INTEREST}

The authors declare no conflict of interest.

\section{ACKNOWLEDGEMENTS}

This work was funded by grants from the NIH (GM099853, NS077958, HD052707) to DW. AEA was partially supported by an NIH training grant (T32GM008136), and ALC was partially supported by NIH training grants (T32GM007055, T32 GM007267). Funding: DW: GM099853, NS077958, HD052707. ALC: T32GM007055, T32GM007267. AEA: T32GM008136. 
1 Geng X, Oliver G: Pathogenesis of holoprosencephaly J Clin Invest 2009; 119 1403-1413.

2 Muenke M, Beachy PA: Genetics of ventral forebrain development and holoprosencephaly. Curr Opin Genet Dev 2000; 10: 262-269.

3 Leoncini E, Baranello G, Orioli IM et al: Frequency of holoprosencephaly in the International Clearinghouse Birth Defects Surveillance Systems: searching for population variations. Birth Defects Res 2008; 82: 585-591.

4 Rubenstein JL, Beachy PA: Patterning of the embryonic forebrain. Curr Opin Neurobiol 1998; 8: 18-26.

5 Golden JA: Holoprosencephaly: a defect in brain patterning. J Neuropathol Exp Neurol 1998; 57: 991-999.

6 Briscoe J, Therond PP: The mechanisms of Hedgehog signalling and its roles in development and disease. Nat Rev Mol Cell Biol 2013; 14: 416-429.

7 Ingham PW, Nakano Y, Seger C: Mechanisms and functions of Hedgehog signalling across the metazoa. Nat Rev Genet 2011; 12: 393-406.

8 Roessler E, Belloni E, Gaudenz K et al: Mutations in the C-terminal domain of Sonic Hedgehog cause holoprosencephaly. Hum Mol Genet 1997; 6: 1847-1853.

9 Roessler E, Belloni E, Gaudenz K et al: Mutations in the human Sonic Hedgehog gene cause holoprosencephaly. Nat Genet 1996; 14: 357-360.

10 Nanni L, Ming JE, Bocian $\mathrm{M}$ et al: The mutational spectrum of the sonic hedgehog gene in holoprosencephaly: $\mathrm{SHH}$ mutations cause a significant proportion of autosomal dominant holoprosencephaly. Hum Mol Genet 1999; 8: 2479-2488.

11 Chiang C, Litingtung Y, Lee E et al: Cyclopia and defective axial patterning in mice lacking Sonic hedgehog gene function. Nature 1996; 383: 407-413.

12 Rallu M, Machold R, Gaiano N, Corbin JG, McMahon AP, Fishell G: Dorsoventral patterning is established in the telencephalon of mutants lacking both Gli3 and Hedgehog signaling. Development 2002; 129: 4963-4974.

13 Aoto K, Nishimura T, Eto K, Motoyama J: Mouse GLI3 regulates Fgf8 expression and apoptosis in the developing neural tube, face, and limb bud. Dev Biol 2002; 251: 320-332.

14 Tole S, Ragsdale CW, Grove EA: Dorsoventral patterning of the telencephalon is disrupted in the mouse mutant extra-toes. Dev Biol 2000; 217: 254-265.

15 El-Jaick KB, Powers SE, Bartholin L et al: Functional analysis of mutations in TGIF associated with holoprosencephaly. Mol Genet Metab 2007; 90: 97-111.

16 Gripp KW, Wotton D, Edwards MC et al: Mutations in TGIF cause holoprosencephaly and link NODAL signalling to human neural axis determination. Nat Genet 2000; 25: 205-208.

17 Powers SE, Taniguchi K, Yen W et al: Tgif1 and Tgif2 regulate Nodal signaling and are required for gastrulation. Development 2010; 137: 249-259.

18 Taniguchi K, Anderson AE, Sutherland AE, Wotton D: Loss of Tgif function causes holoprosencephaly by disrupting the Shh signaling pathway. PLOS Genet 2012; 8 : e1002524.

19 Bartholin L, Powers SE, Melhuish TA, Lasse S, Weinstein M, Wotton D: TGIF inhibits retinoid signaling. Mol Cell Biol 2006; 26: 990-1001.

20 Jin JZ, Gu S, McKinney P, Ding J: Expression and functional analysis of Tgif during mouse midline development. Dev Dyn 2006; 235: 547-553.

21 Mar L, Hoodless PA: Embryonic fibroblasts from mice lacking Tgif were defective in cel cycling. Mol Cell Biol 2006; 26: 4302-4310.

22 Shen J, Walsh CA: Targeted disruption of Tgif, the mouse ortholog of a human holoprosencephaly gene, does not result in holoprosencephaly in mice. Mol Cell Biol 2005; 25: 3639-3647.

23 Bertolino E, Reimund B, Wildt-Perinic D, Clerc R: A novel homeobox protein which recognizes a TGT core and functionally interferes with a retinoid-responsive motif. J Biol Chem 1995; 270: 31178-31188.
24 Hyman CA, Bartholin L, Newfeld SJ, Wotton D: Drosophila TGIF proteins are transcriptional activators. Mol Cell Biol 2003; 23: 9262-9274.

25 Melhuish TA, Wotton D: The Tgif2 gene contains a retained intron within the coding sequence. BMC Mol Biol 2006; 7: 2.

26 Wotton D, Knoepfler PS, Laherty CD, Eisenman RN, Massague J: The Smad transcriptional corepressor TGIF recruits mSin3. Cell Growth Differ 2001; 12: 457-463.

27 Melhuish TA, Wotton D: The interaction of C-terminal binding protein with the Smad corepressor TG-interacting factor is disrupted by a holoprosencephaly mutation in TGIF. J Biol Chem 2000; 275: 39762-39766.

28 Melhuish TA, Gallo CM, Wotton D: TGIF2 interacts with histone deacetylase 1 and represses transcription. J Biol Chem 2001; 276: 32109-32114.

29 Wotton D, Lo RS, Lee S, Massague J: A Smad transcriptional corepressor. Cell 1999; 97: 29-39.

30 Zerlanko BJ, Bartholin L, Melhuish TA, Wotton D: Premature senescence and increased TGFbeta signaling in the absence of Tgif1. PLoS One 2012; 7: e35460.

31 Massague J, Seoane J, Wotton D: Smad transcription factors. Genes Dev 2005; 19 : 2783-2810.

32 Schmierer B, Hill CS: TGFbeta-SMAD signal transduction: molecular specificity and functional flexibility. Nat Rev Mol Cell Biol 2007; 8: 970-982.

33 Pramfalk C, Melhuish TA, Wotton D, Jiang ZY, Eriksson M, Parini P: TG-interacting factor 1 acts as a transcriptional repressor of sterol O-acyltransferase 2. J Lipid Res 2014; 55: 709-717.

34 Wotton D, Lo RS, Swaby LA, Massague J: Multiple modes of repression by the smad transcriptional corepressor TGIF. J Biol Chem 1999; 274: 37105-37110.

35 Yang Y, Hwang CK, D'Souza UM, Lee SH, Junn E, Mouradian MM: Tale homeodomain proteins Meis2 and TGIF differentially regulate transcription. J Biol Chem 2000; 275 20734-20741.

36 Hayashi S, Lewis P, Pevny L, McMahon AP: Efficient gene modulation in mouse epiblast using a Sox2Cre transgenic mouse strain. Mech Dev 2002; 119(Suppl 1): S97-S101.

37 Bjerke GA, Yang CS, Frierson HF, Paschal BM, Wotton D: Activation of Akt signaling in prostate induces a TGFbeta-mediated restraint on cancer progression and metastasis. Oncogene 2014; 33: 3660-3667.

38 Bjerke GA, Hyman-Walsh C, Wotton D: Cooperative transcriptional activation by KIf4, Meis2, and Pbx1. Mol Cell Biol 2011; 31: 3723-3733.

39 Abbasi AA, Paparidis Z, Malik S et al: Human intronic enhancers control distinct subdomains of Gli3 expression during mouse CNS and limb development. BMC Dev Biol 2010; 10: 44.

40 Coy S, Caamano JH, Carvajal J, Cleary ML, Borycki AG: A novel Gli3 enhancer controls the Gli3 spatiotemporal expression pattern through a TALE homeodomain protein binding site. Mol Cell Biol 2011; 31: 1432-1443.

41 Visel A, Blow MJ, Li Z et al: ChIP-seq accurately predicts tissue-specific activity of enhancers. Nature 2009; 457: 854-858.

42 Aoto K, Shikata Y, Imai H et al: Mouse Shh is required for prechordal plate maintenance during brain and craniofacial morphogenesis. Dev Biol 2009; 327: $106-120$.

43 Nomura M, Li E: Smad2 role in mesoderm formation, left-right patterning and craniofacial development. Nature 1998; 393: 786-790.

44 De La Cruz JM, Bamford RN, Burdine RD et al: A loss-of-function mutation in the CFC domain of TDGF1 is associated with human forebrain defects. Hum Genet 2002; 110: 422-428.

45 Roessler E, Ouspenskaia MV, Karkera JD et al: Reduced NODAL signaling strength via mutation of several pathway members including $\mathrm{FOXH} 1$ is linked to human heart defects and holoprosencephaly. Am J Hum Genet 2008; 83: 18-29.

Supplementary Information accompanies this paper on European Journal of Human Genetics website (http://www.nature.com/ejhg) 